\title{
21
}

\section{Time analysis of fault propagation in SDH-based ATM networks}

\author{
K. Van Doorselaere (INTEC), K. Struyve (INTEC), C. Brianza \\ (ITALTEL), P. Demeester (INTEC) \\ Department of Information Technology (INTEC/IMEC), \\ University of Ghent \\ St-Pietersnieuwstraat 41, B-9000 Ghent, Belgium, \\ Tel : + 32926433 24, Fax : + 3292643593 , \\ Email :kvdoor@intec.rug.ac.be
}

\begin{abstract}
This paper provides a detailed analysis of the fault propagation in an SDH-based ATM network from a network view. Both transient and steady state conditions are considered and their influence on the triggering of recovery mechanisms.
\end{abstract}

\section{Keywords}

Faultpropagation, recovery triggering, SDH, ATM

\section{INTRODUCTION}

Telecommunications networks are subject to random failures caused by natural disasters, wear out, overload, human error, etc. and intentional failures caused by sabotage. A failure affects transmission and/or switching facilities which in turn disrupts residential and business user traffic. Preventive actions such as fire safety plans, armoured cables, etc. try to reduce the failure occurrence frequency. Reactive actions on the other hand heal the network after failure occurrence. Reactive actions can be categorised into three phases, i.e. fault detection, notification and localisation actions, recovery actions and repair actions. Recovery 
involves, preferable autonomous, rerouting of disrupted traffic bypassing the failed network facilities, whereas repair involves substitution and mending of failed network facilities.

Consider an SDH-based ATM network transporting ATM traffic via SDH connections. A root or primary failure such as a cable cut event causes an avalanche of consequent or secondary failures such as disrupted ATM Virtual Paths (VP). More precisely ITU-T recommendation M.20 defines a failure as 'the termination of the ability of an item to perform a required function' and a fault as 'the inability of an item to perform a required function'.

Operation and maintenance functions implemented in the distributed network elements detect these faulty items resulting from the corresponding failures, and notify downstream and upstream network elements as well as TMN. Based on this possibly overwhelming number of fault notifications TMN tries to localise the primary fault cause.

Standard bodies have defined maintenance signals and fault detection and notification mechanisms among others for SDH (ETS 300 417, 1996), (ITU-T G.783, 1993), (ITU-T G.783-draft, 1996) and ATM (ITU-T I.610, 1995), (ITU-T Q19/13, 1997). Similar concepts and mechanisms for WDM are currently under study. These recommendations define the individual fault detection and notification functional components from a network element view but do not provide a network level view. This paper addresses therefore the timing of fault detection and notification from a network perspective rather than from a network element view and pays special attention to fault propagation across network layers, i.e. Physical Section (PS) layer, Regenerator Section (RS) layer, Multiplex Section (MS) layer, Higher Order Path (HOP) layer, ATM Virtual Path (VP or Avp) layer, for an SDH-based ATM network. Clarification of these mechanisms is not a goal in itself but rather a pre-requisite to study among others survivability strategies for multi-layer networks (Nederlof, Struyve., O'Shea., Misser., Du., Tamayo, 1995). A possible survivability strategy for an SDH-based ATM network, known as 'interworking at the lowest layer' (ACTS-PANEL project, 1997) (Demeester et al., 1997), involves recovery at the SDH layer in case of a physical or SDH layer primary failure and recovery at the ATM layer in case of an ATM layer primary failure. Note that an ATM layer failure such as an ATM switch breakdown cannot be recovered at the lower SDH layer, and hence demands recovery at the ATM layer. Although ATM layer recovery may also resolve physical and SDH layer failures, this strategy uses SDH layer recovery since less entities with a coarser granularity have to be rerouted and thus a higher recovery speed is expected. Correct and fast fault propagation plays clearly an important role in this strategy. Indeed, to minimise service impact recovery must be triggered and completed, either at the SDH layer or at the ATM layer dependent on the primary failure, in the range of milliseconds to seconds.

The rest of the paper is organised as follows: in the first section the elementary fault detection and propagation mechanisms are explained, used to notify the 
down- and upstream network elements of the failure; in the second section these mechanisms are applied on a SDH based ATM network scenario: two different fault cases are presented and analysed in detail and some issues are pointed out; in the third section some generalised conclusions are derived and finally the influence of the fault detection and propagation mechanisms on the triggering of recovery mechanisms is explained. Used abbreviations are explained in an appendix.

\section{CONSIDERATIONS}

As mentioned above, a root failure causes an avalanche of secondary failures, notified in the downstream network elements. The detection of these secondary failures is performed in atomic functions within the network elements. An anomaly is the first indication that there is something wrong in the network. Some examples of anomalies are : the detection of the Out Of Frame (OOF) condition within the adaptation sink function between the Physical Section and Regenerator Section layer (PS/RS_A_Sk function), the detection of the Out of Cell Delineation (OCD) condition within the adaptation sink function between the SDH VC4 layer and the ATM VP layer (S4/Avp_A_Sk function), the reception of a single all-ONEs signal or a single invalid AU-pointer within the adaptation sink function between the Multiplex Section layer and the VC4 layer (MS/S4_A_Sk function). If there is sufficient density of anomalies for a short period of time (defined as detection time) a defect is declared (defect filter F1, see figure 4). The detection time depends on the defect type and may even vary for a specific defect type. For instance the Loss Of Signal defect (dLOS) detection time is dependent among others on the power level of the input signal before the failure (e.g. the fibre cut) and on the receiver technology being used. APD/SAW filter receivers could detect LOS in about $50 \mu \mathrm{s}$, but some experiments have proved that pin-fet/PLL receivers may need several milliseconds before detecting dLOS when the input signal before the break is at maximum power level. Thus sometimes it may also happen that the Loss Of Frame defect (dLOF) is detected before dLOS within an equipment. The dependency of the fault propagation mechanism on the detected defects and their required detection times makes that this paper distinguishes two realistic and important fault scenarios, supposing a fibre cut, which resolves in a quick or slow dLOS-detection.

The detection of an anomaly doesn't imply any consequent action within an atomic function (consequent action filter F2). Thus while an atomic function is integrating an anomaly into a defect, it passes through the input data under all conditions. As such, an STM-frame filled with corrupted bytes, will present this invalid information to all downstream atomic functions more or less at the same time (see note about transfer delays later in this section). When an atomic function detects a defect, the incoming data signal is no longer forwarded and an Alarm Indication Signal (AIS) (this is an all-ONEs data stream in SDH) is propagated towards the next atomic function. Each downstream atomic function passes 
through these all-ONEs until this atomic function declares a defect and inserts new AIS-signals (either new all-ONEs, either VP-AIS cells). Remark that, complementary to the all-ONEs signal, the defect condition is also forwarded to the next atomic function by a Characteristic Information Server Signal Fail (CI_SSF) in case an adaptation sink function detects the defect and by an Adapted Information Trail Signal Fail (AI_TSF) in case a trail termination sink function detects the defect. ${ }^{1}$ In this next atomic function, possibly not performing AISdetection, the CI_SSF and AI_TSF represent the defect condition and can thereby possibly, among other consequent actions, control AIS-insertion. As such, the insertion of all-ONES in the Administrative Unit (AU)-pointer within the adaptation source function between the Multiplex Section layer and the SDH VC-4 layer (MS/S4_A_So function) is not controlled by the presence of all-ONES in the VC4 path layer data signal, but is controlled by the CI_SSF emitted by the MS/S4_A_Sk function as a consequence of the Administrative Unit Loss Of Pointer defect (AUdLOP) or Administrative Unit Alarm Indication Signal defect (AUdAIS) detection (ETS 300 417-3-1, 1996). Within two frames (read : "at most the next second frame") from the reception of the CI_SSF signal, the MS/S4_A_So function will replace the outgoing AU pointer with an all-ONES pointer (AUU-AIS).

Each atomic function correlates its detected defects (fault cause filter F3) and the result, a fault cause, is reported to the Equipment Management Function (EMF) of the network element (ETS 300417,1996 ). When a fault cause persists for X seconds (default value for $X=2.5 \pm 0.5 \mathrm{~s}$ ) in the failure filter F4 (see figure 4), it is reported to TMN as a failure (ETS 300417,1996 ). In TMN these failures are correlated to identify the root failure. A correct correlation in the atomic functions requires thus that the fault cause filter $\mathrm{F} 3$ in each atomic function rejects secondary defects and holds back the primary defect. As such, the LOF fault cause (cLOF) will not being reported to the EMF on an incoming AI_TSF, indicating that dLOS was detected. Some of the secondary defects are autonomously cleared on receipt of AIS, SSF or TSF. For example, defects as MS-DEG, MS-EXC, AUdLOP are being cleared on incoming all-ONEs signalling, indicating that the failure was already noticed more upstream. Some atomic functions, not detecting the allONEs, clear some of their defects on the reception of a CI_SSF or AI_TSF signal. For example, the S4_TT_Sk function, not performing all-ONEs detection, will clear its defects (dUNEQ, dTIM, etc...) on an incoming CI_SSF. In a similar way, a correct correlation in the S4/Avp_A_Sk requires that the LCD fault cause (cLCD) is cleared on an incoming AI_TSF, indicating an ATM physical layer trail signal fail condition. Remark that this is not yet standardised.

For a detailed time analysis of the fault propagation also transfer delays should be taken into account. Within an equipment transfer delays may be introduced by

\footnotetext{
${ }^{1}$ CI_SSF and AI_TSF are functional signals and realised often as either hardware or software signals and are thus implementation dependent.
} 
buffers (e.g. pointer buffer, mapping and demapping buffers, jitter reduction buffers), switch fabric, frame alignment circuits, encoders and decoders, series/parallel and parallel/series convertors and other equipment specific processes. For a VC-4 the transfer delay within an equipment is about $10 \mu \mathrm{s}$, which is quite low in comparison with the frame repetition time $(125 \mu \mathrm{s})$ and therefore neglected in this paper. Another transfer delay is added in the fibres, depending on the length of the fibre : e.g. a signal needs $250 \mu$ s to propagate through a fibre of $50 \mathrm{~km}$.

The next section discusses in detail the fault detection and propagation mechanism for an SDH based ATM network scenario. Two fault scenarios are considered : $\mathrm{dLOS}$ is detected quick or slow after a fibre cut.

\section{QUICK AND SLOW LOSS OF SIGNAL DEFECT DETECTION}

\subsection{Network scenario}

Figure 1 presents a multilayer (ATM-VP layer over SDH HOP layer) network scenario, consisting of some basic SDH and ATM equipment, which is connected by bidirectional lines (i.e. a fibre in each direction). In the scenario a fibre cut has been supposed (root failure). The high level sequence of events in steady state conditions is itemised below.

1. LOS is detected in the regenerator.

2. MS-AIS is generated and sent to DXC-AU_1.

3. MS-RDI is generated and sent in the upstream direction.

4. MS-AIS is escalated to AU-AIS, which is sent to DXC-AU_2.

5. AU-AIS is forwarded to ATM-VPXC_1.

6. HP-RDI is generated and sent in upstream direction.

7. VP-AIS is sent downstream for each VP connection supported by the affected SDH path.

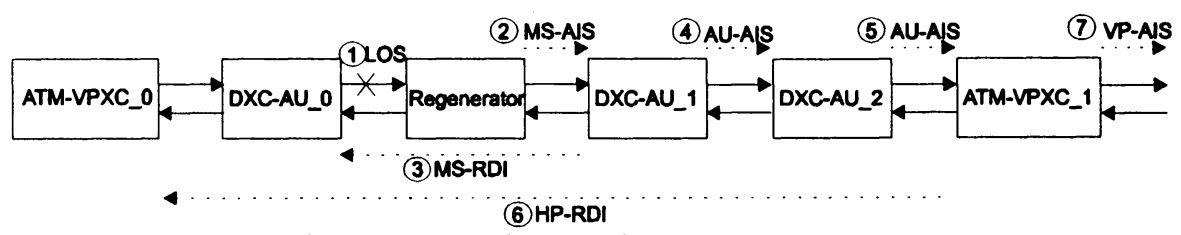

Figure 1 SDH based ATM network scenario.

\subsection{Quick LOS defect detection}

In this fault scenario it is assumed that the fibre cut leads to a quick LOS detection in the regenerator (and thus before the detection of other defects in the network). The fault propagation mechanism is illustrated in figure $2 a$, while the timing aspects are represented in figure $2 b$. 

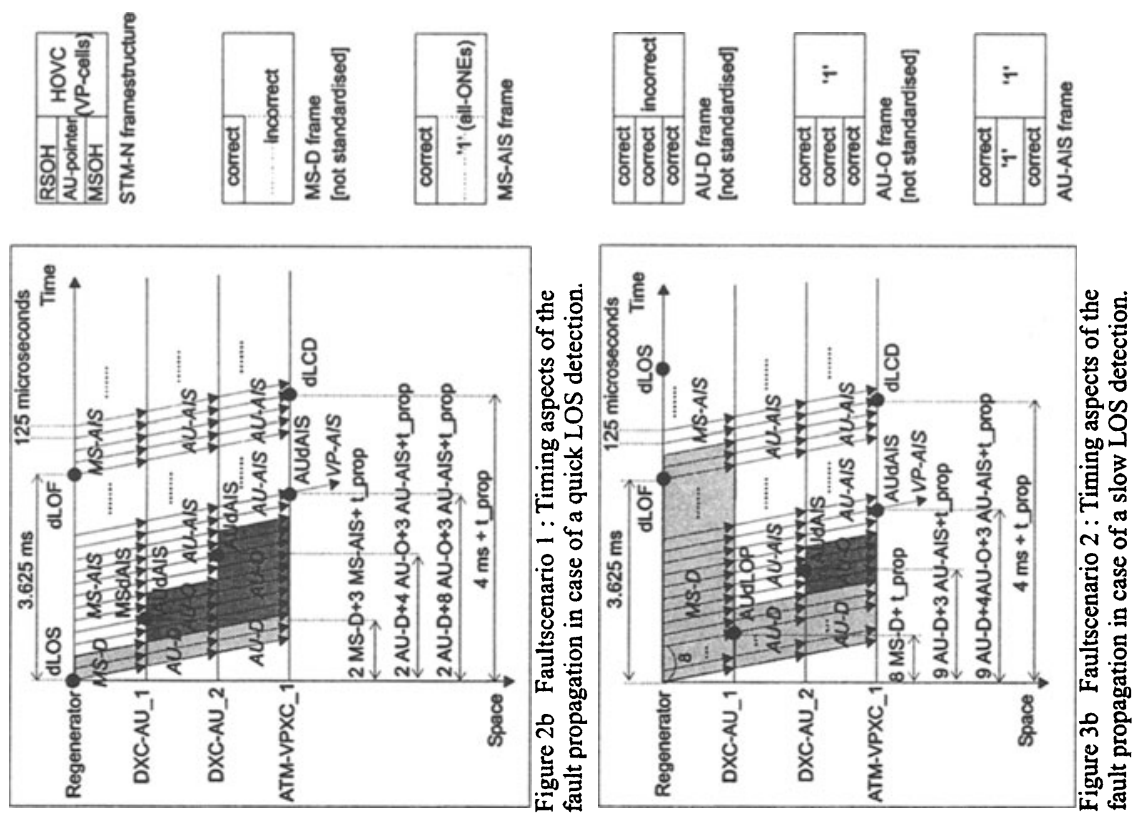

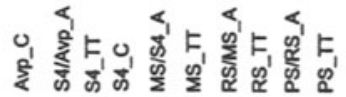

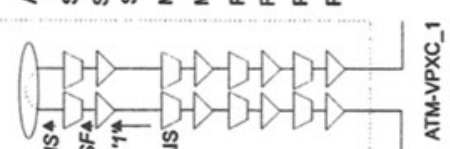
की

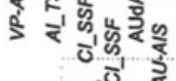

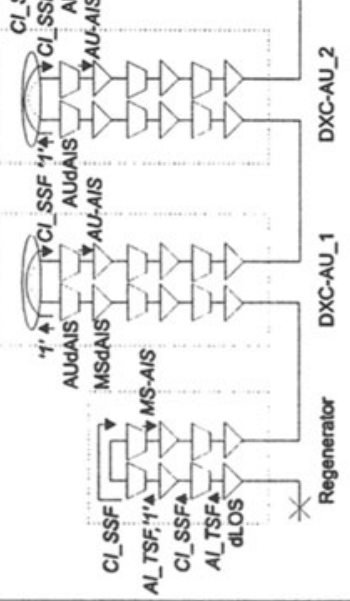

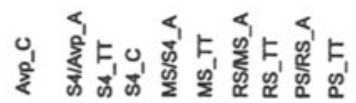

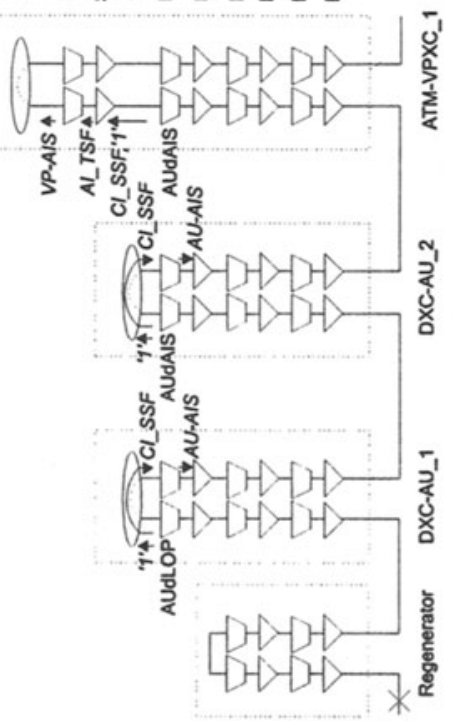


The Trail Termination sink function in the Physical Section layer (PS_TT_Sk function) of the regenerator notices that the physical input signal is no longer valid and declares the dLOS-defect. In the meantime this atomic function generates $0 / 1$ bits in a random way (depending on the hardware technology) and inserts them in the downstream direction. Each downstream atomic function passes through this digitised noise until a defect is detected and AIS signals (all-ONEs/VP-AIS cells) are inserted. Upon the declaration of dLOS an AI_TSF is activated in the PS_TT_Sk function towards the PS/RS_A_Sk function. This signal is refreshed in each of the next atomic functions, either as CI_SSF either as AI_TSF and resolves finally in the RS/MS_A_So function within 2 frames into the insertion of MS-AIS towards DXC-AU_1, containing a valid Regenerator Section Overhead (RSOH) and an all-ONEs regenerator payload. Each downstream atomic function passes through these all-ONEs until an AIS defect or other local defect is detected and new AIS signals (all-ONEs/VP-AIS cells) are inserted. In summary, after the fibre cut the regenerator outputs 2 frames downstream, containing a valid RSOH but digitised noise in the regenerator payload (later on referred to as MS-D frames), before generating MS-AIS signals.

The digitised noise in the MS-D frames initiates in the DXC-AU_1 the detection of anomalies, such as the MS BIP-24N violations in the MS_TT_Sk function and invalid pointers in the MS/S4_A_So function. The MS-AIS signalling (containing all-ONEs in the regenerator payload) however suppresses these anomalies and results instead in the declaration of two AIS-defects : the MS_TT_Sk function declares the MSdAIS defect after having received for $X$ ( $X$ is in the range 3 to 5) consecutive frames ' 111 ' in bits $6,7,8$ of byte $\mathrm{K} 2$, while the MS/S4_A_Sk declares the AUdAIS defect after having received for 3 consecutive frames ' 11111111 ' in the $\mathrm{H} 1$ and $\mathrm{H} 2$ bytes (AU-pointer bytes). Within 2 frames after the MSdAISdetection the upstream direction is notified by the insertion of an MS-RDI signal. Within 2 frames after the declaration of the AIS defect (MSdAIS, AUdAIS), the MS_TT_Sk function respectively the MS/S4_A_Sk function renew the all-ONEs stream. In the meantime the incoming all-ONEs-stream is passed through transparently. The detection of AUdAIS activates however also a S4_CI_SSF towards the MS/S4_A_So function, which adds within 2 frames an all-ONEs AUpointer to the Higher Order Virtual Containers (HOVC). Meanwhile normal AUpointers are written to the HOVCs in the generated STM-frames. In summary, after the root failure the DXC-AU_1 outputs 2 AU-D frames (no standardised definition) (containing the same noise in the HOVC as the MS-D frames, but a correct AU-pointer and new Section Overhead (SOH), 4 AU-O frames (no standardised definition) (containing the same all-ONEs in the HOVC as the incoming MS-AIS, but a new valid AU-pointer and new SOH) and only then AUAIS (with a new all-ONEs HOVC, an all-ONEs AU-pointer and correct SOH).

In DXC-AU_2 no anomalies, except for AUdAIS, are observed because of the renewed overhead of the STM-frames in DXC-AU_1. When the AU-AIS frames enter DXC-AU_2, 3 consecutive frames with the all-ONEs AU-pointers are 
required to declare AUdAIS and 2 more frames to output AU-AIS. The AU-AIS signal is thus again delayed for 4 frames (compared to the HOVC payload in the STM-frame) and the DXC-AU_1 will output meanwhile 4 AU-O frames.

In ATM-VPXC_1 no Physical -, Regenerator - or Multiplex Section anomalies are noticed. AUdAIS is again detected after 3 consecutive AU-AIS frames. Making the summation, 2 AU-D frames +4 AU-O frames +4 AU-O frames +3 AU-AIS frames entered ATM-VPXC_1 since the fibre cut until AUdAIS declaration. The AUdAIS detection time depends thus on the number of intermediate AU-pointer processing functions.

The detected AUdAIS activates a CI_SSF and this signal, refreshed in the S4_TT_Sk function as AI_TSF, causes thereby in the S4/Avp_A_Sk function the insertion of VP-AIS, the first as soon as possible and then every second one. In the S4_TT_Sk function the CI_SSF suppresses the detection of defects and generates within 2 frames a HP-RDI to notify the upstream network elements. In the S4/Avp_A_Sk function the AI_TSF suppresses the fault cause cLCD but not the defect dLCD. After receiving thus for $4 \mathrm{~ms}$ invalid VP-cell headers, containing random bits or all-ONEs, dLCD is declared, activating also VP-AIS cells.

As already highlighted, the detection time of AUdAIS in ATM-VPXC_1 depends on the number $n$ of intermediate AU-pointer processing functions before this network element. Consider therefore the network scenario presented in figure $2 \mathrm{c}$, consisting of a regenerator and $n$ DXC-AUs before ATM-VPXC_1.

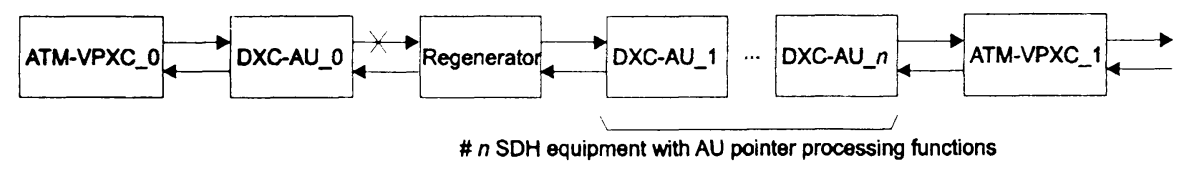

$n>7:$ dLCD detected before AUdAIS
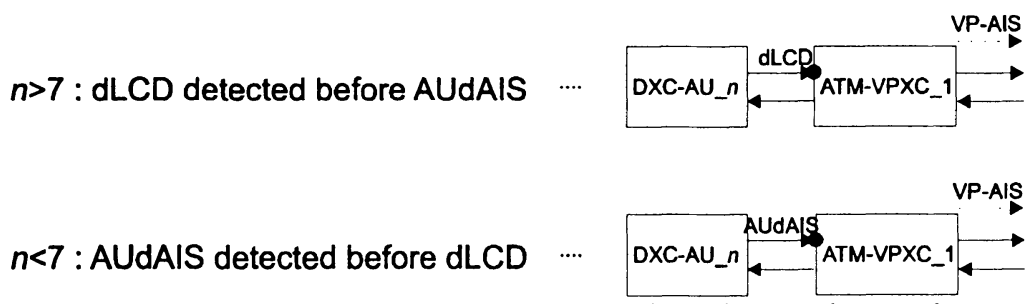

Figure 2c The dependency of the dLCD and AUdAIS detection on the number $n$ of SDH equipment with AU-pointer processing functions in case of a quick LOS.

Reusing the reasoning above, 2 AU-D frames $+n^{*} 4$ AU-O frames +3 AU-AIS frames entered ATM-VPXC_1 since the fibre cut until the detection of the AUdAIS defect. If $n>7$, the defect dLCD is detected before the defect AUdAIS in ATM-VPXC_1 (remark that the defect dLCD is detected wihin $4 \mathrm{~ms}$, equalling 32 frames). Supposing $n>7$, it is the defect dLCD that activates the insertion of the VP-AIS cells in ATM-VPXC_1. When finally AUdAIS is detected, this sends a CI_SSF/AI_TSF towards the S4/Avp_A_Sk function, which clears the fault cause 
cLCD. Because the fault causes are integrated on a $X$ seconds basis in the EMF of the network element, the correct failure will be passed to TMN.

\subsection{Slow LOS defect detection}

In this fault scenario the receiver technology notices very slowly the fibre cut, and thus secondary defects such as dLOF will be detected before the primary defect dLOS.

As a consequence of the slow reacting receiver technology, the PS_TT_Sk function assumes that the input signal is still valid and generates thus incorrect decoded bits in the downstream direction. As such, the PS/RS_A_Sk function declares, while hunting for the A1A2-bit pattern to align the frames, after 5 frames with incorrect A1A2-bytes an OOF-anomaly, which results $3 \mathrm{~ms}$ later in the defect dLOF. Within 2 frames after dLOF-declaration, MS-AIS-signals are inserted to inform DXC-AU_2. When finally dLOS is detected, the generated AI_TSF clears in the PS/RS_A_Sk function the fault cause cLOF. In summary, after the fibre cut the regenerator outputs 30 MS-D frames (containing a correct RSOH but incorrect decoded bits in the regenerator payload) before generating MS-AIS frames.

The incorrect regenerator payload of the MS-D frames causes also in the other atomic functions in the chain the detection of anomalies. In the MS/S4_A_Sk of DXC-AU 1 this results in the declaration of the defect AUdLOP after the detection of 8 consecutive invalid AU-pointer-anomalies. Within 2 frames after AUdLOP the MS/S4_A_Sk inserts all-ONEs in the downstream direction. At the same moment results the CI_SSF, emitted by the MS/S4_A_Sk, in the MS/S4_A_So in the writing of all-ONEs AU-pointers to the incoming HOVCs (containing the all-ONEs) and generates thus AU-AIS signals. In conclusion, after the fibre cut the DXC-AU_1 has output 9 AU-D frames (containing the same incorrect HOVC payload as the MS-D frames but with a new correct AU-pointer and $\mathrm{SOH}$ ) before generating AU-AIS signals. Thus it is the detection of a defect in a downstream equipment (i.e. AUdLOP) that initiates the insertion of AISsignals. When finally MS-AIS signals reach the DXC-AU_1, both AIS-defects are detected again : the detected MSdAIS suppresses thereby the MS BIP-24N violations and generates also within 2 frames in the upstream direction an MS-RDI, while the detected AUdAIS clears the AUdLOP defect.

For the fault detection and propagation mechanism downstream of DXC-AU_1 can be referred to the fault scenario with a quick LOS. As thus, after the fibre cut 9 AU-D frames + 4 AU-O frames + 3 AU-AIS frames must enter ATM-VPXC_1 before declaring the AUdAIS defect. The AUdAIS is thus detected first in ATMVPXC_1 and activates the insertion of the VP-AIS cells.

Considering now again the network scenario of figure 2c, ATM-VPXC_1 will detect AUdAIS after it received 9 AU-D frames $+(n-1) * 4$ AU-O frames +3 AUAIS frames since the fibre cut. Thus if $n>6$, the defect dLCD-defect is detected before the defect AUdAIS in the ATM-VPXC. 
$n>6:$ dLCD detected before AUdAIS

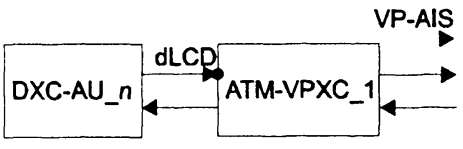

$n<6:$ AUdAIS detected before dLCD $\quad . .$.

Figure 3c The dependency of the dLCD and AUdAIS detection on the number $n$ of SDH equipment with AU-pointer processing functions in case of a slow LOS.

\section{GENERALISED CONCLUSIONS}

The fault scenarios analysed in the previous subsections show that during transient conditions after failure occurrence the network sometimes behaves in a strange way. Such strange behaviour manifests itself principally in the following events:

- detection of defects within the downstream equipment quite before the detection of the primary defect (e.g. AUdLOP detection in DXC-AU_1 before dLOF detection in the upstream regenerator - see 2.3).

- anticipated manifestation of an upper layer defect which is detected before the correlated lower layer defect within the same equipment (e.g. dLCD defect detection before the AUdAIS defect detection in an ATM VPXC - see 2.2 and 2.3).

Table 1 summarises for the explained fault scenarios the first detected defect in the network and explains the dependency of the AUdAIS and ALCD defect detection in ATM-VPXC_1 on the number $n$ of AU-pointer processing functions in front of ATM-VPXC_1.

Table 1: Side effects during the transient period of the defect detection and propagation process in an SDH based ATM network.

\begin{tabular}{cccc}
\hline $\begin{array}{c}\text { Fault } \\
\text { scenario }\end{array}$ & $\begin{array}{c}\text { First defect } \\
\text { detected in } \\
\text { the network }\end{array}$ & $\begin{array}{c}\text { x frames entered ATM- } \\
\text { VPXC_l since root } \\
\text { failure till AUdAIS- } \\
\text { detection, with } x \text { : }\end{array}$ & $\begin{array}{c}\text { dLCD detected } \\
\text { before AUdAIS in } \\
\text { ATM-VPXC_1 if } n>\end{array}$ \\
\hline quick LOS & dLOS & $2+n^{*} 4+3$ & 7 \\
slow LOS & AUdLOP & $9+(n-1)^{* 4}+3$ & 6 \\
\hline
\end{tabular}

TMN however will never notice these side during the transient period of the defect detection and propagation, due to the fault cause integration for $\mathrm{X}$ seconds $(\mathrm{X}: 2.5$ $\pm 0.5)$ in the EMF before reporting them as a failure to TMN. 
After the root failure is repaired, valid data will be transmitted again through the network. Only when the declared defects in each atomic function are cleared, it will stop the insertion of the all-ONEs signal and pass through the valid data. In consequence, the defect clearing is performed in a serial way and takes thus much longer than the defect propagation. As a result, supposing a temporary failure, the defect clearing will never catch up the defect propagation. Due to the fault cause integration of $\mathrm{X}$ seconds, no failure notification reports will be generated on a temporary fault.

\section{GUIDELINES}

In case the recovery strategy is defined as 'interworking at the lowest layer' (ACTS-PANEL project, 1997) (Demeester et al., 1997), a distributed recovery mechanism in e.g. the ATM VP layer (distributed restoration/protection) should only be triggered on ATM VP layer primary failures or on non recoverable server layer failures and provide thus non redundant protection. For the network scenario of figure 2c, this implies that a fibre cut in the span of DXC-AUs should be recovered within this span and not trigger ATM VP layer recovery mechanisms in the ATM-VPXCs. As the presented fault scenarios made clear, the ATM-VPXCs will always detect a defect in the range of a few milliseconds, whereas the SDH recovery will require at least $50 \mathrm{~ms}$ (protection). The ATM-VPXC may thus not trigger an ATM-VP layer recovery mechanism on the simple detection of a defect and requires thus more defined conditions. Steady state fault causes can provide thereby useful information : e.g. the steady state fault cause cLCD in an ATMVPXC indicates that the S4/Avp_A_So function of the upstream ATM network element has failed, which can not be recovered in the lower SDH layer and demands thus recovery in the ATM-VP layer. To assure however that the transient conditions are elapsed, a reasonable persistency time of the fault causes is required, to be defined on the basis of the values suggested by the presented fault scenarios. Remark that this recovery strategy requires also other mechanisms, besides the use of the fault causes, to control a correct triggering of the recovery protocols. These mechanisms are currently under study in the PANEL-project. Figure 4 illustrates the most relevant fault management filters used in this paper and proposes some parts of a triggering architecture for the 'interworking at the lowest layer'-strategy.

\section{ACKNOWLEDGEMENT}

This work has been partly funded by the ACTS project AC205 - PANEL : "Protection Across Network Layers". During the study of this topic, M.P.J. Vissers (Lucent Technologies Network Systems Nederland) has provided valuable information about the fault management mechanisms. 


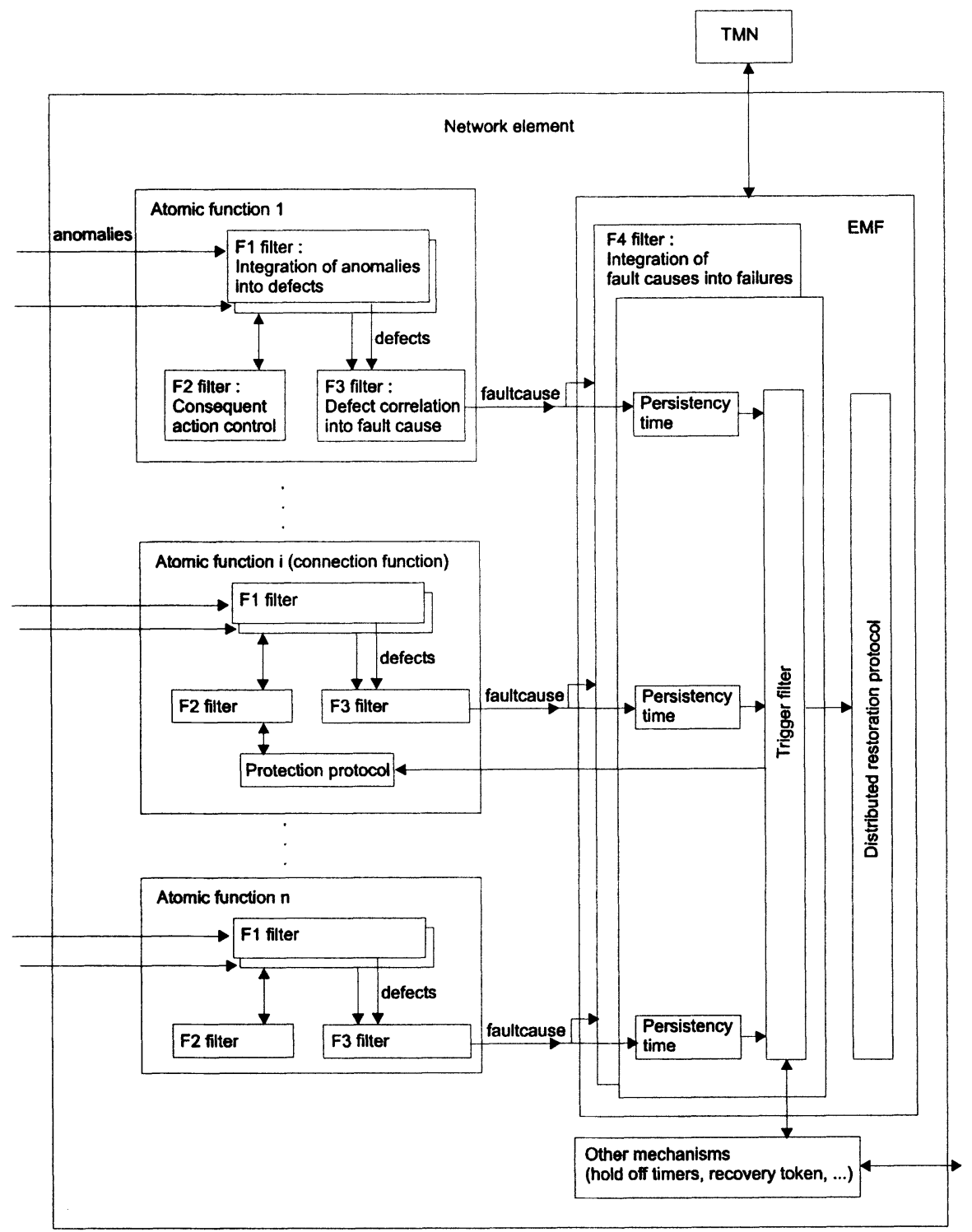

Figure 4 Recovery triggering architecture within a NE.

\section{APPENDIX}

This appendix explains used abbreviations and acronyms.

- Layers : PS (Physical Section); RS (Regenerator Section); MS (Multiplex Section); S4 (SDH VC-4 path layer); VP or Avp (ATM Virtual Path layer). 
- STM-frame structure : AU (Administrative Unit); HOVC (Higher Order Virtual Container); MSOH (Multiplex Section Overhead); RSOH (Regenerator Section Overhead); SOH (Section Overhead).

- Atomic functions : X/Y_A_Sk : Adaptation Sink function between client layer $\mathrm{Y}$ and server layer $\mathrm{X} ; \mathrm{X} / \mathrm{Y} \_\mathrm{A}$ _So : Adaptation Source function between client layer $\mathrm{Y}$ and server layer $\mathrm{X} ; \mathrm{X}$ _C : Connection function in layer X; X_TT_Sk : Trail Termination Sink function in layer $X ; X_{-}$TT_So : Trail Termination Source function in layer X.

- Anomalies : OCD (Out Of Cell Delineation); OOF (Out Of Frame).

- Defects : dAIS (Alarm Indication Signal defect); dDEG (degraded defect); dLCD (Loss of Cell Delineation defect); dLOF (Loss Of Frame defect); dLOP (Loss Of Pointer defect); dLOS (Loss Of Signal defect); dPLM (Payload Mismatch defect); dRDI (Remote Defect Indicator defect); dTIM (Trace Identifier Mismatch defect); dUNEQ (unequipped defect); Exc (Excessive error defect).

- Alarm signals AIS (Alarm Indication Signal); AI_TSF (Adapted Information Trail Signal Fail); CI_SSF (Characteristic Information Server Signal Fail); RDI (Remote Defect Indicator).

\section{REFERENCES}

ACTS-PANEL project (1997). Deliverable D2a : Overall Network ProtectionVersion 1.

Demeester et al., (1997) PANEL - Protection across network layers. NOC '97, European Conference on Networks and Optical Communications, Antwerp, Belgium.

ETSI Recommendation (1996). ETS 300417 : Transmission and Multiplexing (TM); Generic functional requirements for Synchronous Digital Hierarchy (SDH) equipment.

ITU-T Recommendation (1993). G.783 : Characteristics of Synchronous Digital Hierarchy (SDH) Equipment Functional Blocks.

ITU-T Recommendation (1995). I.610 : B-ISDN Operation and Maintenance Principles and Functions.

ITU-T Recommendation (1996). G.783-draft : Characteristics of Synchronous Digital Hierarchy (SDH) Equipment Functional Blocks.

ITU-T Q.19/13 Rapporteur's Meeting (16-20 June 1997). AIS in the ATM Network Functional Model. Turin, Italy.

Nederlof L., Struyve K., O'Shea C., Misser H., Du Y., Tamayo B. (September 1995) End-to-end survivable broadband Networks. IEEE Communications Magazine. 